\title{
Erratum to: Society position statement
}

\section{Canadian Cardiovascular Society/Canadian Anesthesiologists' Society/Canadian Heart Rhythm Society joint position statement on the perioperative management of patients with implanted pacemakers, defibrillators, and neurostimulating devices}

\author{
Jeff S. Healey, MD • Richard Merchant, MD • Chris Simpson, MD • \\ Timothy Tang, MD $\cdot$ Marianne Beardsall, MN/NP $\cdot$ Stanley Tung, MD • \\ Jennifer A. Fraser, RN • Laurene Long, RN • Janet M. van Vlymen, MD · \\ Pirjo Manninen, MD · Fiona Ralley, MBBCh $\cdot$ Lashmi Venkatraghavan, MD · \\ Raymond Yee, MD • Bruce Prasloski, MD • Shubhayan Sanatani, MD • \\ François Philippon, MD
}

Published online: 12 May 2012

(C) Canadian Anesthesiologists' Society and Canadian Cardiovascular Society 2012

Erratum to: Can J Anesth/J Can Anesth (2012)

59:394-407

DOI 10.1007/s12630-012-9678-8

In the published manuscript, under the subheading

"Industry resources", the link for Boston Scientific's online CRD reference database is incorrect and should read: http://www.bostonscientific.com/templatedata/imports/ HTML/CRM/Product_Performance_Resource_Center/index. shtml.
The online version of the original article can be found under doi:10.1007/s12630-012-9678-8.

\section{J. S. Healey, MD ( $\square)$}

Population Health Research Institute, McMaster University,

Room C3-121, David Braley CVSRI, 237 Barton St East,

Hamilton, ON L8L 2X2, Canada

e-mail: Jeff.Healey@phri.ca

R. Merchant, MD · S. Tung, MD · B. Prasloski, MD .

S. Sanatani, MD

University of British Columbia, Vancouver, BC, Canada

C. Simpson, MD · J. M. van Vlymen, MD

Queen's University, Kingston, ON, Canada

T. Tang, MD

University of Calgary, Calgary, AB, Canada

M. Beardsall, MN/NP

Southlake Regional Health Centre, Newmarket, ON, Canada

M. Beardsall, MN/NP

University of Toronto, Toronto, ON, Canada

\author{
J. A. Fraser, RN \\ Peterborough Regional Cardiac Device Clinic, Peterborough, \\ ON, Canada \\ L. Long, RN \\ Hamilton Health Sciences, Hamilton, ON, Canada \\ P. Manninen, MD · L. Venkatraghavan, MD \\ University Health Network, University of Toronto, Toronto, ON, \\ Canada \\ F. Ralley, MBBCh $\cdot$ R. Yee, MD \\ University of Western Ontario, London, ON, Canada \\ F. Philippon, MD \\ Université Laval, Quebec City, QC, Canada
}

